\title{
The Central Amygdala Nucleus is Critical for Incubation of Methamphetamine Craving
}

\author{
Xuan Li*,', Tamara Zeric', Sarita Kambhampati', Jennifer M Bossert' and Yavin Shaham*,' \\ 'Behavioral Neuroscience Branch, Intramural Research Program of NIDA/NIH, Baltimore, MD, USA
}

\begin{abstract}
Cue-induced methamphetamine seeking progressively increases after withdrawal but mechanisms underlying this 'incubation of methamphetamine craving' are unknown. Here we studied the role of central amygdala (CeA), ventral medial prefrontal cortex (vmPFC), and orbitofrontal cortex (OFC), brain regions implicated in incubation of cocaine and heroin craving, in incubation of methamphetamine craving. We also assessed the role of basolateral amygdala (BLA) and dorsal medial prefrontal cortex (dmPFC). We trained rats to selfadminister methamphetamine ( 10 days; $9 \mathrm{~h} / \mathrm{day}, 0.1 \mathrm{mg} / \mathrm{kg} /$ infusion) and tested them for cue-induced methamphetamine seeking under extinction conditions during early ( 2 days) or late ( $4-5$ weeks) withdrawal. We first confirmed that 'incubation of methamphetamine craving' occurs under our experimental conditions. Next, we assessed the effect of reversible inactivation of CeA or BLA by GABAA + GABAB receptor agonists (muscimol + baclofen, $0.03+0.3 \mathrm{nmol}$ ) on cue-induced methamphetamine seeking during early and late withdrawal. We also assessed the effect of muscimol + baclofen reversible inactivation of VmPFC, dmPFC, and OFC on 'incubated' cue-induced methamphetamine seeking during late withdrawal. Lever presses in the cue-induced methamphetamine extinction tests were higher during late withdrawal than during early withdrawal (incubation of methamphetamine craving). Muscimol + baclofen injections into CeA but not BLA decreased cue-induced methamphetamine seeking during late but not early withdrawal. Muscimol+baclofen injections into dmPFC, vmPFC, or OFC during late withdrawal had no effect on incubated cue-induced methamphetamine seeking. Together with previous studies, results indicate that the CeA has a critical role in incubation of both drug and non-drug reward craving and demonstrate an unexpected dissociation in mechanisms of incubation of methamphetamine vs cocaine craving.

Neuropsychopharmacology (2015) 40, 1297-1306; doi: I0. I038/npp.20 14.320; published online 7 January 2015
\end{abstract}

\section{INTRODUCTION}

Drug addiction is associated with high rates of relapse during abstinence (Jaffe, 1990; Wikler, 1973). Exposure to drug-associated cues and contexts is a common trigger for drug relapse, even during prolonged abstinence periods (O'Brien et al, 1992). We and other investigators previously demonstrated that cue-induced cocaine and heroin seeking progressively increases after withdrawal from drug selfadministration in rats (Grimm et al, 2001; Neisewander et al, 2000; Shalev et al, 2001). We termed this phenomenon as incubation of drug craving (Grimm et al, 2001; Lu et al, 2004b). Subsequently, we showed that incubation of drug craving also occurs in rats with a history of methamphetamine self-administration (Shepard et al, 2004; Theberge et al, 2013). During the past decade, a number of studies have identified neurobiological and pharmacological mechanisms of incubation of cocaine craving and, to a lesser

\footnotetext{
*Correspondence: X Li or Dr Y Shaham, Behavioral Neuroscience Branch, Intramural Research Program of NIDANIH, 25I Bayview Blvd, Baltimore, MD 21224, USA, Tel: +I 443740 2723, Fax: +I 443 740 2723, E-mail: anna.li@nih.gov or yshaham@intra.nida.nih.gov Received 12 September 2014; revised 4 November 2014; accepted 5 November 2014; accepted article preview 5 December 2014
}

degree, incubation of heroin craving (Loweth et al, 2014; Marchant et al, 2013; Pickens et al, 2011; Wolf and Ferrario, 2010). In contrast, the mechanisms underlying incubation of methamphetamine craving have not been explored.

Based on previous findings with heroin and cocaine, in the present study we assessed the roles of central and basolateral amygdala (CeA and BLA, respectively), ventral and dorsal medial prefrontal cortex (vmPFC and dmPFC, respectively), and orbitofrontal cortex (OFC) in incubation of methamphetamine craving. We chose CeA because previous studies showed that inhibition of CeA neuronal activity decreases incubation of cocaine and sucrose craving in the self-administration procedure (Lu et al, 2005; Lu et al, 2007; Uejima et al, 2007), as well as incubation of morphine craving in the conditioned place preference procedure (Li et al, 2008). We studied the role of BLA because of its established role in conditioned drug effects and cueinduced reinstatement of drug seeking (Everitt et al, 1999; See, 2002). Recent evidence also implicates BLA-to-nucleus accumbens (NAc) projections in incubation of cocaine craving (Lee et al, 2013). In addition, we previously demonstrated that reversible inactivation of vmPFC (comprised of infralimbic and ventral part of prelimbic cortices) and lateral OFC decreases incubation of cocaine and heroin craving, respectively (Fanous et al, 2012; Koya et al, 2009). 
Furthermore, the dmPFC (comprised of anterior cingulate and dorsal part of prelimbic cortices) has been established as a critical brain region for cue-induced reinstatement of drug seeking (Bossert et al, 2013; Feltenstein and See, 2008). Recent studies also implicate the projection from this brain area to NAc in incubation of cocaine craving (Ma et al, 2014).

Here we used the GABAA and GABAB receptor agonists muscimol + baclofen (McFarland and Kalivas, 2001) to reversibly inactivate CeA, BLA, vmPFC, dmPFC, or OFC to determine their causal roles in incubation of methamphetamine craving. We first determined whether reversible inactivation of a given brain area decreases the 'incubated' cue-induced drug seeking in the extinction tests during late withdrawal (4-5 weeks, herein termed 1 month). Then we followed up on positive findings by determining the effect of reversible inactivation of the brain area on 'non-incubated' cue-induced drug seeking during early withdrawal (day 2). We found that reversible inactivation of the CeA (but not the other brain areas) during late but not early withdrawal decreased cueinduced methamphetamine seeking, indicating a critical role of the $\mathrm{CeA}$ in incubation of methamphetamine craving.

\section{MATERIALS AND METHODS}

\section{Subjects}

We used Male Sprague-Dawley rats (Charles River, total $n=199$ ), weighing $300-350 \mathrm{~g}$ prior to surgery and $325-375 \mathrm{~g}$ at the start of the drug self-administration procedure; we maintained the rats under a reverse 12:12-h light/dark cycle with food and water freely available. We kept the rats two per cage prior to surgery and then housed them individually after surgery. We performed the experiments in accordance with the National Institutes of Health Guide for the Care and Use of Laboratory Animals (eighth edition), under the protocols approved by the Animal Care and Use Committee. We excluded 59 rats due to failure of catheter patency, health-related issues, failure to acquire stable methamphetamine self-administration, or cannula misplacement. The number of rats reported herein refers to rats included in the statistical analyses.

\section{Intravenous Surgery}

See Supplementary Online Material.

\section{Intracranial Surgery}

See Supplementary Online Material.

\section{Intracranial Injections}

See Supplementary Online Material.

\section{Apparatus}

See Supplementary Online Material.

\section{Methamphetamine Self-Administration Training}

We used a training procedure previously described by Theberge et al (2013) and Krasnova et al (2014). We brought the rats to the self-administration room on their first day of training and housed them chronically in the self-administration chambers. We trained the rats to self-administer methamphetamine $9 \mathrm{~h}$ per day (three 3-h sessions, separated by $1 \mathrm{~h}$ between sessions) under a fixedratio-1 with a 20-s timeout reinforcement schedule. We dissolved methamphetamine in saline, and the rats selfadministered methamphetamine at a dose of $0.1 \mathrm{mg} / \mathrm{kg} /$ infusion over $3.5 \mathrm{~s}(0.10 \mathrm{ml} /$ infusion $)$. We trained the rats for 10 sessions over a 14-day period (off day every third day) in order to prevent loss of body weight during the training phase. (Note: methamphetamine-trained rats lose about 4-8g after each day of training and regain the lost weight during the off day (Krasnova et al, 2014; Theberge et al, 2013)). During training, active lever presses led to the delivery of a methamphetamine infusion and a compound 5-s tone-light cue (the tone and light modules (Med Associates) were located above the active lever). During the 20-s timeout, we recorded the non-reinforced lever presses. The daily training sessions started at the onset of the dark cycle and began with the extension of the active lever and the illumination of a red house light that remained on for the duration of the session. At the end of each 3-h session, the red house light was turned off and the active lever retracted. The training data from Exps. 1-3 are described in Figure 1.

\section{Withdrawal Phase}

During the withdrawal phase, we housed the rats individually in the animal facility and handled them 3-4 times per week.

\section{Extinction Test}

We conducted all extinction tests immediately after the onset of the dark cycle. For Exps. 2 and 3, we started the extinction test session $15 \mathrm{~min}$ after intracranial injections. The sessions began with the extension of the active lever and the illumination of the red house light, which remained on for the duration of the session. Active lever presses during testing (the operational measure of cue-induced drug seeking in incubation of craving studies ( $\mathrm{Lu}$ et al, 2004b; Pickens et al, 2011)) resulted in contingent presentations of the tone-light cue, previously paired with methamphetamine infusions but not the drug.

\section{Food Self-Administration}

See Supplementary Online Material.

\section{Exp. 1: Incubation of Methamphetamine Craving After Withdrawal}

The goal of this initial experiment was to determine that 'incubation of methamphetamine' is observed under our experimental conditions. We trained 18 rats to self-administer methamphetamine as described above. We tested 8 rats after 2 withdrawal days and 10 rats after 30-35 withdrawal days (termed herein 1 month) for cue-induced methamphetamine seeking under extinction conditions (Extinction test) in a 2-h session. We counterbalanced the two group of

Neuropsychopharmacology 
rats based on their methamphetamine infusions during selfadministration training.

\section{Exp. 2: Effect of Amygdala Inactivation on Incubation of Methamphetamine Craving}

We performed intravenous surgeries on the eight groups of rats (total $n=71$ ) and implanted them with bilateral guide cannulas $1 \mathrm{~mm}$ above the CeA or BLA. We then trained them for methamphetamine self-administration in four independent runs. In the first two runs, we determined the effect of reversible inactivation of CeA and BLA on extinction responding after 1 month of withdrawal; in the last two runs, we assessed the inactivation effect after 2 withdrawal days (Figure 3a). (Note: because of the longterm nature of 'incubation of craving' studies, our research strategy is to first determine the reversible inactivation effect during late withdrawal and then follow-up on positive findings with assessment on withdrawal day 2 , in order to determine whether the effect of reversible inactivation is selective to the late withdrawal 'incubated' response or reflects a general time-independent effect on cue-induced drug seeking ( $\mathrm{Lu}$ et al, 2007).)

On either withdrawal day 2 or after 1 month of withdrawal, we injected different groups of rats bilaterally with vehicle or muscimol + baclofen $(0.03 \mathrm{nmol}+0.3 \mathrm{nmol} / 0.5 \mu \mathrm{l} / \mathrm{side})$ into CeA or BLA 15 min before the 3-h extinction tests. The number of rats per group after exclusion due to misplacements was: CeA-Day 2-vehicle, $n=11$; CeA-Day 2-muscimol+ baclofen, $n=9$; $\quad$ BLA-Day 2-vehicle, $n=10 ; \quad$ BLA-Day 2 -muscimol + baclofen, $n=7$; CeA-1 month-vehicle, $n=9$; CeA-1 month-muscimol + baclofen, $n=11$; BLA-1 monthvehicle, $n=7$; and BLA-1 month-muscimol + baclofen, $n=7$.

Finally, to ensure that the effect of $\mathrm{CeA}$ inactivation by muscimol + baclofen on extinction responding during the late withdrawal test (see Results section) was not due to motor deficits, we trained five rats from the vehicle group for food self-administration (see Supplementary Online Material) after they completed CeA injections and extinction tests on withdrawal day 2 . We trained them for 7 days $(1 \mathrm{~h} /$ day $)$ and then injected them with vehicle or muscimol + baclofen $(0.03 \mathrm{nmol}+0.3 \mathrm{nmol} / 0.5 \mu \mathrm{l} /$ side $)$ into CeA, 15 min before the 1-h food self-administration session on the eighth and ninth day; we counterbalanced the order of the vehicle and muscimol + baclofen injections.

\section{Exp. 3: Effect of Prefrontal Cortex Inactivation on Incubation of Methamphetamine Craving}

We performed intravenous surgeries on the six groups of rats $(n=43)$ and implanted them with bilateral guide cannulas $1 \mathrm{~mm}$ above vmPFC, dmPFC, or OFC. We then trained them for methamphetamine self-administration in three independent runs. After 1 month of withdrawal, we injected the rats bilaterally with vehicle or muscimol + baclofen $(0.03 \mathrm{nmol}+0.3 \mathrm{nmol} / 0.5 \mu \mathrm{l} /$ side $)$ into vmPFC, dmPFC, or OFC $15 \mathrm{~min}$ before the 3 -h extinction tests. The number of rats per group after exclusion due to misplacements was: vmPFC-vehicle, $n=9$; vmPFC-muscimol + baclofen, $n=7$; dmPFC-vehicle, $n=7$; dmPFC-muscimol + baclofen, $n=5$; OFC-vehicle, $n=7$; and OFC-muscimol + baclofen, $n=8$.
Statistical Analysis

See Supplementary Online Material.

\section{RESULTS}

\section{Exp. 1: Incubation of Methamphetamine Craving}

Self-administration training. The rats increased their number of methamphetamine infusions over days $\left(\mathrm{F}_{9,153}=\right.$ $12.8, p<0.01)$. Analysis of lever presses demonstrated that active but not inactive lever increased over days (Lever $x$ Day interaction, $\mathrm{F}_{9,153}=3.6, p<0.01$; Figure 1a).

Extinction tests. Cue-induced methamphetamine seeking in the extinction tests was higher after 1 month of withdrawal than after 2 days, demonstrating incubation of methamphetamine craving' under our experimental conditions. The statistical analysis of total active lever presses showed a significant interaction between Withdrawal period and Lever $\left(\mathrm{F}_{1,15}=12.4, p<0.01\right)$. Analysis of the time course of active lever presses showed a significant

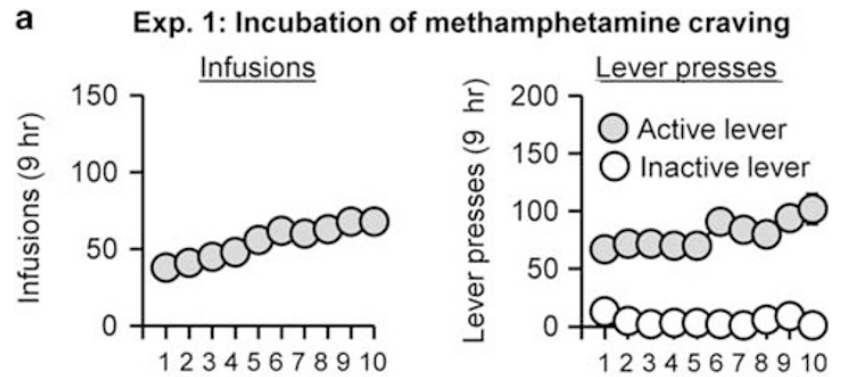

b $\quad$ Exp. 2: Amygdala inactivation
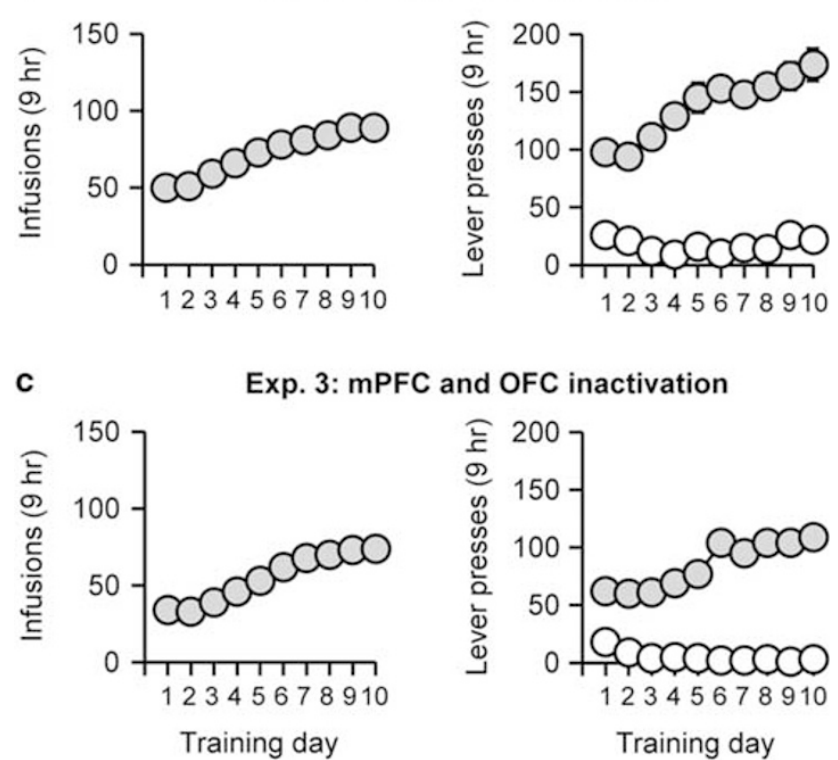

Figure I Methamphetamine self-administration training. Data are mean \pm SEM of the methamphetamine infusions (Left column) and leverpresses (Right column) during the 10 9-h daily self-administration sessions (3 3-h sessions separated by I h) for Exp. I (total $n=\mid 8$ ), Exp. 2 (total $n=7 I$ ), and Exp. 3 (total $n=43$ ). During training, active lever presses were reinforced on an FRI 20-s timeout reinforcement schedule, and methamphetamine infusions were paired with a 5-s tone-light cue. 
a

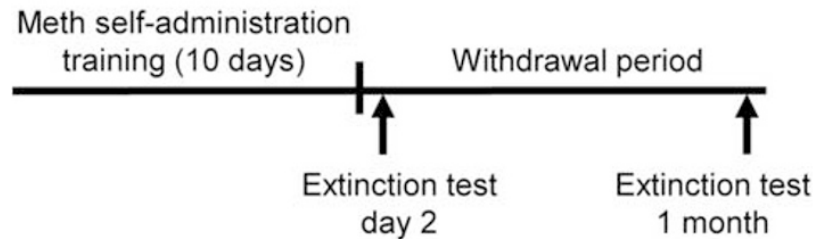

Figure 2 Time-dependent increases in cue-induced methamphetamine seeking after withdrawal (incubation of methamphetamine craving). (a) Timeline of the experiment. ( $b$ and $c$ ) Mean \pm sem total lever presses and time course of lever presses during the extinction tests after 2 withdrawal days and after I month of withdrawal. During testing, leverpresses led to contingent presentations of the tone-light cue previously paired with methamphetamine infusions during training but did not result in an infusion of methamphetamine. Data are mean \pm SEM of the lever presses on the previously active lever and on the inactive lever during the extinction sessions. *Different from day $2, p<0.01, n=8-10$ per group. Meth $=$ methamphetamine

interaction between Withdrawal period and Session time $\left(\mathrm{F}_{3,48}=3.0, p<0.01\right.$; Figure 2).

Exp. 2: Effect of Amygdala Inactivation on Incubation of Methamphetamine Craving

Self-administration training. The rats increased their number of methamphetamine infusions over days $\left(\mathrm{F}_{9,630}=79.8, p<0.01\right)$. Analysis of lever presses showed that active but not inactive lever increased over days (Lever $\times$ Day interaction, $\mathrm{F}_{9,630}=12.3, p<0.01$; Figure $1 \mathrm{~b}$ ).

Extinction tests. Muscimol + baclofen injections into the CeA but not BLA decreased cue-induced methamphetamine seeking after 1 month of withdrawal but not after 2 days. Analysis of active lever presses (inactive lever presses as a covariate) showed a significant interaction of Withdrawal period $\times$ Drug $\quad\left(\mathrm{F}_{1,35}=5.8, p<0.05\right)$. Analysis of time courses of active lever presses showed a significant triple interaction of Withdrawal period $\times$ Drug $\times$ Session time $\left(\mathrm{F}_{2,72}=10.6, p<0.01\right)$. In contrast, muscimol + baclofen injections into the BLA had no effect on cue-induced methamphetamine seeking after 1 month of withdrawal or after 2 days. Analysis of active lever presses (inactive lever presses as a covariate) showed a significant effect of Withdrawal period $\left(\mathrm{F}_{1,26}=19.3, p<0.01\right)$ but no other main effects of interactions ( $p$-values $>0.1)$. Analysis of the time course of active lever presses showed a significant effect of

session time $\left(\mathrm{F}_{2,54}=70.4, p<0.01\right)$ and Withdrawal period $\left(\mathrm{F}_{1,27}=20.9, p<0.01\right)$ but no other main effects of interactions ( $p$-values $>0.1$ ). Additionally, muscimol + baclofen injections outside the CeA or BLA (misplacements) had no effect on extinction responding during early ( 2 days) or late (1 month) withdrawal (Figure 3). Furthermore, muscimol + baclofen injections into either the CeA or BLA had no effect on inactive lever presses, which were significantly lower than active lever presses; inactive lever presses were somewhat higher after 1 month of withdrawal (range 10-22 lever presses per $3 \mathrm{~h}$ in the different groups) than after 2 days (range 4-20 lever presses). The latter effect, which is commonly observed in our incubation of craving studies (Airavaara et al, 2011; Lu et al, 2004a; Theberge et al, 2013), likely reflects response generalization after prolonged withdrawal (Shalev et al, 2002).

Finally, to rule out that the effect of CeA inactivation is due to motor deficits, we trained rats $(n=5)$ to selfadminister palatable or preferred food pellets (Calu et al, 2014) and determined the effect of vehicle or muscimol + baclofen CeA injections on ongoing food-reinforced responding. We found that muscimol + baclofen injection into CeA had no effect on food self-administration. The number of food pellets and active lever presses (mean \pm SEM), respectively, during the 1 -h test sessions was $127 \pm 17$ and $379 \pm 97$ after vehicle injections and $120 \pm 18$ and $395 \pm 150$ after muscimol + baclofen injections.

\section{Exp. 3: Effect of Prefrontal Cortex Inactivation on Incubation of Methamphetamine Craving}

Self-administration training. The rats increased their number of methamphetamine infusions over days $\left(\mathrm{F}_{9,378}=119.0, p<0.01\right)$. The analysis of lever presses showed that active but not inactive lever increased over days (Lever $\times$ Day interaction, $\mathrm{F}_{9,378}=30.6, \quad p<0.01$; Figure 1c).

Extinction tests. Muscimol + baclofen injections into vmPFC, dmPFC, or OFC had no effect on 'incubated' cue-induced methamphetamine seeking after 1 month of withdrawal. Analysis of active lever presses (inactive lever presses as a covariate) showed no significant effects of Drug (vehicle, muscimol + baclofen) for $\operatorname{vmPFC}\left(\mathrm{F}_{1,13}=0.1\right.$, $p>0.1)$ dmPFC $\left(\mathrm{F}_{1,9}=0.3, p>0.1\right)$, and $\mathrm{OFC}\left(\mathrm{F}_{1,12}=0.1\right.$, $p>0.1)$. Analysis of time course of active lever presses showed a significant effect of Session time for the three brain areas: vmPFC $\left(\mathrm{F}_{2,28}=12.2, \quad p<0.01\right), \quad \mathrm{dmPFC}$ $\left(\mathrm{F}_{2,20}=22.0, \quad p<0.01\right)$, and $\mathrm{OFC} \quad\left(\mathrm{F}_{2,26}=7.7, \quad p<0.01\right.$; Figure 4).

\section{DISCUSSION}

We used an established reversible inactivation method to study the roles of CeA, BLA, vmPFC, dmPFC, and OFC in incubation of methamphetamine craving. Our first main finding was that reversible inactivation of CeA but not BLA decreased 'incubated' cue-induced methamphetamine seeking after prolonged withdrawal. These results indicate a critical role of $\mathrm{CeA}$ in incubation of methamphetamine craving and extend previous findings on the critical role of 


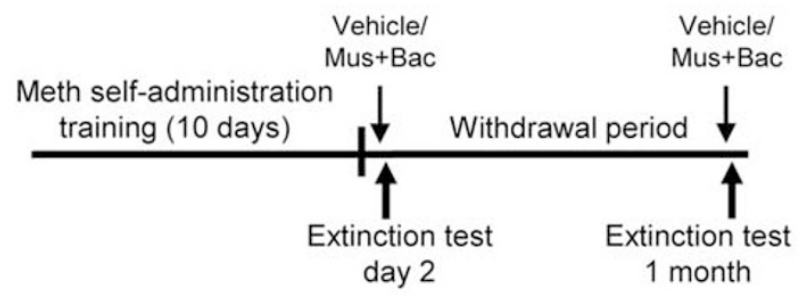

b

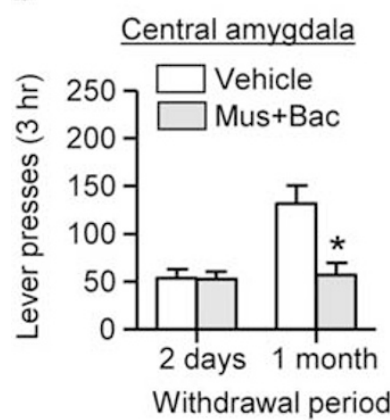

C

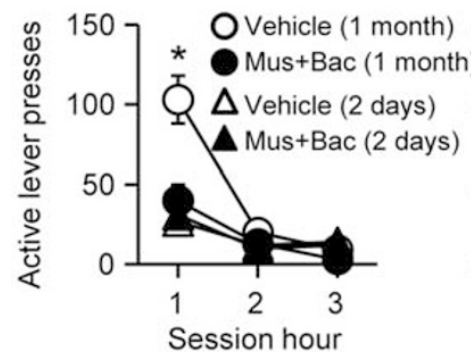

Total responding

\section{Basolateral amygdala}

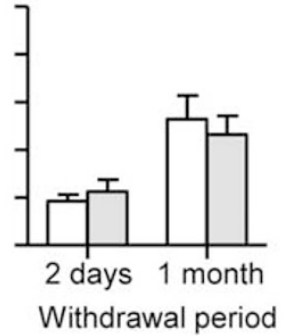

Time course d

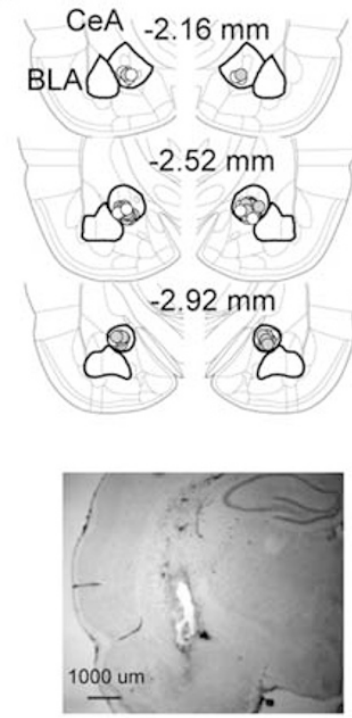

Cannula placements
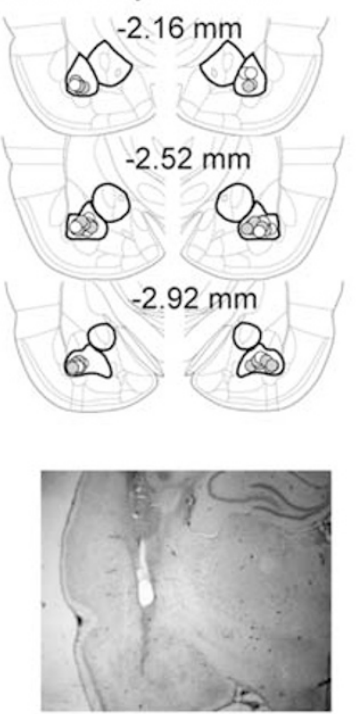

\section{$\underline{\text { CeA or BLA misplacements }}$}

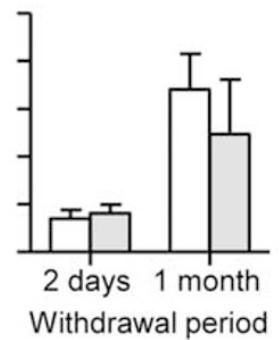

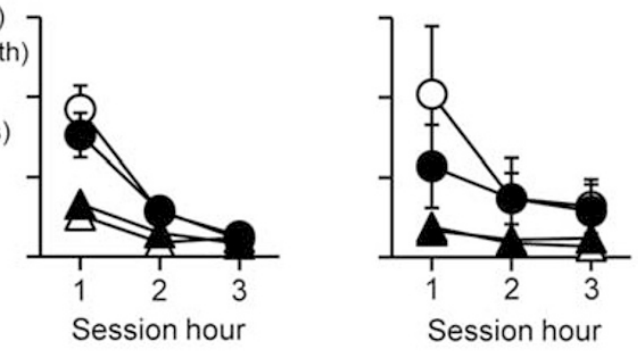

Figure 3 Reversible inactivation of CeA but not BLA decreased cue-induced methamphetamine seeking after prolonged but not early withdrawal. (a) Timeline of the experiment. ( $b$ and c) Extinction tests after 2 withdrawal days and after I month of withdrawal following bilateral injections of vehicle or muscimol + baclofen $(0.03+0.3 \mathrm{nmol} / \mathrm{side})$ : Left: CeA $(n=9-1 \mathrm{I})$. Middle: BLA $(n=7-10)$, Right: misplaced injections $(n=3-8)$. During testing, leverpresses led to contingent presentations of the tone-light cue previously paired with methamphetamine infusions during training. Data are mean $\pm \mathrm{SEM}$ of the responses on the previously active lever during the extinction tests. *Different from vehicle, $p<0.05$. (d) Approximate placement (mm from Bregma, (Paxinos and Watson, 2008)) of injection tips (vehicle: open circles; muscimol + baclofen: closed circles) and representative cannula placements. Meth $=$ methamphetamine. 


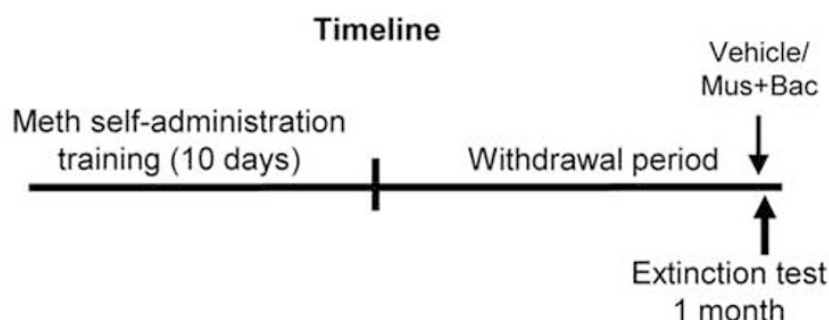

b

Total responding

Ventral mPFC

Dorsal mPFC

Orbitofrontal cortex
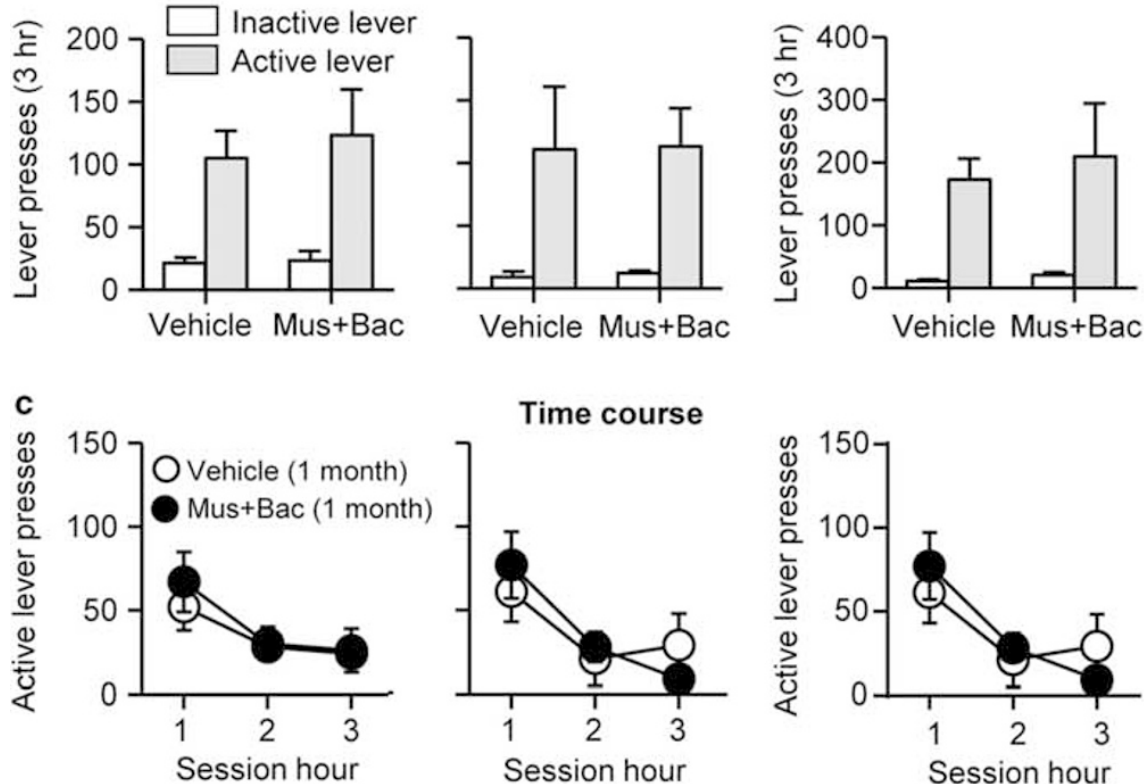

d

Cannula placements

+3.72 mm<smiles>CC12CCCC1C1CCC2C1</smiles>

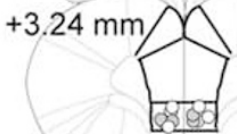
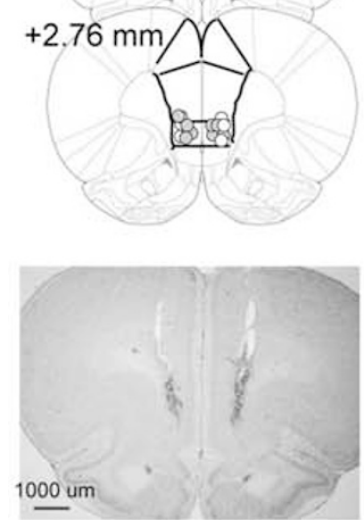

$+3.72 \mathrm{~mm}$
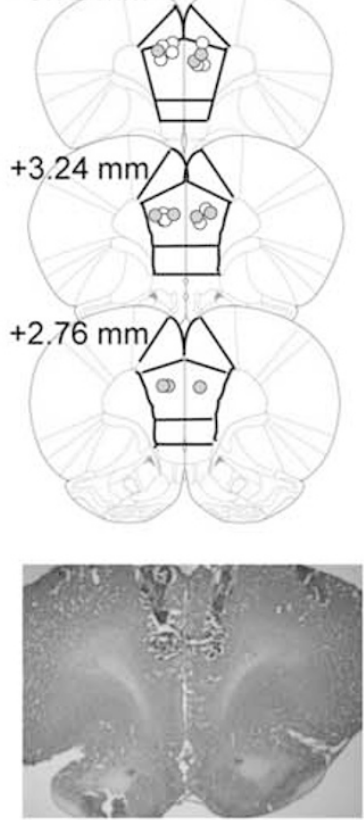

$+4.20 \mathrm{~mm}$

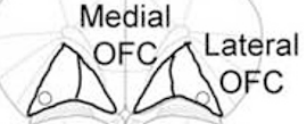

$+3.72 \mathrm{~mm}$

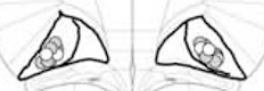

$+3.24 \mathrm{~mm}$

62

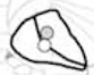

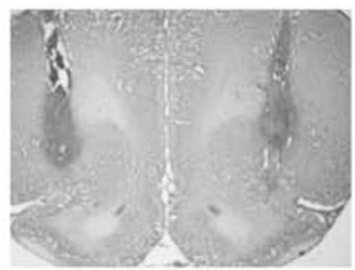


this brain area in incubation of drug and non-drug rewards (Li et al, 2008; Lu et al, 2005; Lu et al, 2007; Uejima et al, 2007). Our second major finding was that reversible inactivation of vmPFC, previously implicated in incubation of cocaine craving (Koya et al, 2009), had no effect on incubation of methamphetamine craving. These results demonstrate an unexpected dissociation in mechanisms of incubation of methamphetamine $v s$ cocaine craving.

\section{Methodological Considerations}

Several methodological issues should be considered in data interpretation. One issue is that the effect of CeA muscimol + baclofen injections is due to non-specific performance deficits. This interpretation is unlikely, because these injections had no effect on lever-presses during early withdrawal or high rate operant responding for food. Another issue is the anatomical specificity of the CeA injections. An interpretation issue in studies using intracranial injections is that behavioral changes might be due to drug diffusion from the injected site into the adjacent sites (Wise and Hoffman, 1992). However, it is unlikely that drug diffusion can account for our data, because muscimol + baclofen injections into BLA or other areas outside the CeA (cannula misplacements) had no effect on 'incubated' methamphetamine craving.

In addition, negative findings in current reversible inactivation studies of BLA and cortical areas should be interpreted with caution. This is because studies investigating the role of other areas (eg, NAc) in drug seeking and motivated behaviors have demonstrated dissociable effects of reversible inactivation manipulations $v s$ dopamine or glutamate receptor antagonists (Bossert et al, 2005; Schmidt et al, 2005; Yun et al, 2004).

Recently, Dong and colleagues showed that silentsynapse-based manipulations of the BLA or dmPFC (prelimbic cortex) projections to accumbens shell or core, respectively, decreased incubated cue-induced cocaine seeking during late withdrawal (Lee et al, 2013; Ma et al, 2014). In contrast, reversible inactivation of BLA or dmPFC with muscimol + baclofen had no effect on incubation of methamphetamine (present study) or cocaine (Koya et al, 2009) craving. However, direct comparison between our results and the results by Dong and colleagues (Lee et al, 2013; Ma et al, 2014) should be made with caution because of several notable differences in experimental procedures. We used extended daily drug access (6-9 h/day) and adultonset drug self-administration, while Dong and colleagues used limited drug access ( $2 \mathrm{~h}$ /day) and juvenile-onset selfadministration. It is well established that extended drug access causes different physiological and behavioral changes than limited drug access (Ahmed and Kenny, 2011; Koob and Le Moal, 2001). Additionally, there is evidence from incubation of reward-craving studies that adult-onset selfadministration is associated with different physiological and behavioral changes than juvenile- and adolescent-onset selfadministration (Counotte et al, 2014; Doherty et al, 2013; Li and Frantz, 2009).

\section{The CeA and Incubation of Reward Craving}

An unexpected finding in our study was that reversible inactivation of the $\mathrm{CeA}$ but not the other brain areas decreased the expression of methamphetamine craving during late withdrawal. This selective effect was unexpected, because previous studies have demonstrated that reversible inactivation (lidocaine or muscimol+baclofen) of $\mathrm{CeA}$, BLA, vmPFC, and dmPFC decrease cue-induced reinstatement of methamphetamine seeking after extinction (Hiranita et al, 2006; Rocha and Kalivas, 2010). Additionally, CeA, BLA, vmPFC, dmPFC, and OFC have critical roles in cue-induced reinstatement of cocaine seeking after extinction (Alleweireldt et al, 2006; Fuchs et al, 2004; Kruzich and See, 2001; McLaughlin and See, 2003; Meil and See, 1997; Pockros et al, 2011). What might account for the general role of multiple cortical and amygdala nuclei in cueinduced reinstatement of methamphetamine seeking after extinction $v s$ the selective role of $\mathrm{CeA}$ in incubation of methamphetamine craving?

One issue is that, in the above studies on cue-induced reinstatement after extinction, the rats were trained for $1-3 \mathrm{~h} /$ day (limited access), while we trained our rats for $9 \mathrm{~h} /$ day (extended access), resulting in escalation of drug intake (Figure 1). As mentioned above, these different drug-access procedures cause different behavioral and brain changes. Another issue is that in the above extinction-reinstatement studies, cue-induced drug seeking was assessed after $\sim 5-10$ withdrawal days, while the cueinduced drug seeking test in our study was performed after 1 month of withdrawal. It is well established that certain drug-induced neuroadaptations, which are critical for incubation of cocaine craving and cue-induced drug seeking, only emerge after prolonged withdrawal (Loweth et al, 2014; Shaham and Hope, 2005; Van den Oever et al, 2010; Wolf and Ferrario, 2010). A third issue is that mechanisms of relapse to drug seeking after forced abstinence $v s$ forced abstinence plus extinction training can be mediated by different substrates (Fuchs et al, 2006; Marchant et al, 2013), possibly due to extinction-induced reversal of drug-induced neuroadaptations (Knackstedt et al, 2010; Sutton et al, 2003).

The present and previous results on the critical role of CeA but not BLA in incubation of drug craving (Pickens et al, 2011) may have implications to the understanding of psychological mechanisms of incubation of reward craving. In this regard, one phenomenon that has been consistently

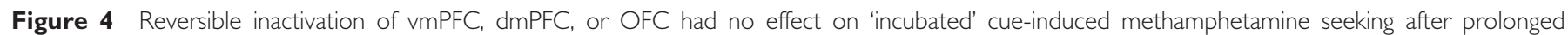

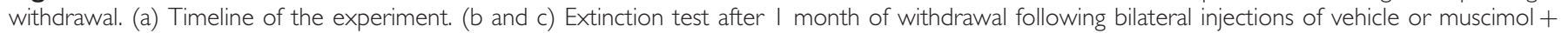

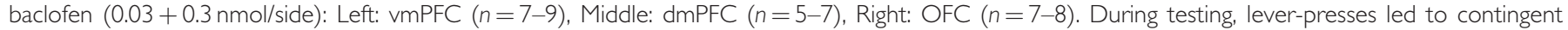

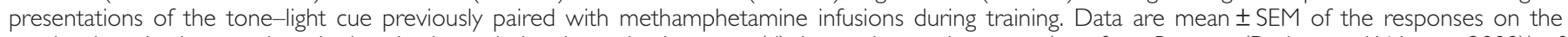

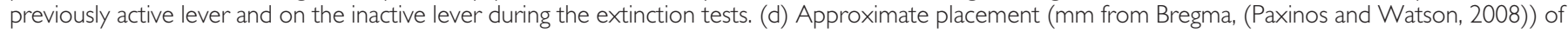
injection tips (vehicle: open circles; muscimol + baclofen: closed circles) and representative cannula placements. 
shown to be sensitive to CeA but not BLA manipulations is Pavlovian-to-Instrumental Transfer (PIT) (Balleine and Killcross, 2006; Hall et al, 2001; Holland and Gallagher, 2003; Mahler and Berridge, 2012), and in particular, the 'general' form of PIT (Corbit and Balleine, 2005), which is thought to represent the general motivational or affective properties of the reward (Corbit and Balleine, 2005). PIT refers to the ability of a Pavlovian conditioned cue, previously paired with non-contingent delivery of a reward, to increase non-reinforced operant responding for the reward after operant training (Estes, 1948; Lovibond, 1983). The magnitude of responding to Pavlovian cues in PIT studies is thought to reflect the motivational potency of reward cues (Mahler and Berridge, 2012; Wyvell and Berridge, 2001). The PIT training procedure (non-contingent pairing of Pavlovian cues with the reward) and the training procedure in incubation studies (contingent pairing of the tone-light or light with the reward) are different. However, in both cases Pavlovian cues can strongly influence subsequent non-reinforced operant responding during testing (Balleine and Killcross, 2006; Shalev et al, 2002). Thus, based on the results from PIT studies described above, we speculate that incubation of drug craving is due to CeA-mediated time-dependent increases in the motivational potency of Pavlovian drugassociated cues after withdrawal.

\section{Implications for Mechanisms of Incubation of Drug Craving for Different Abused Drugs}

We found that reversible inactivation of vmPFC, previously shown to decrease incubated cocaine seeking (Koya et al, 2009), had no effect on incubated methamphetamine seeking. This finding is surprising based on the commonly held notion, derived from numerous studies, that psychostimulant-induced dopamine release in the mesocorticolimbic dopamine system mediates psychostimulant reward (Pierce and Kumaresan, 2006; Wise, 2004) and relapse (Schmidt et al, 2005; Self, 2004). However, our results from the incubation studies are in agreement with previous findings demonstrating that different $\mathrm{mPFC}$ mechanisms contribute to methamphetamine $v s$ cocaine seeking, as assessed in the operant reinstatement procedure (Bossert et al, 2013). Specifically, reversible inactivation of the vmPFC induces reinstatement of cocaine but not methamphetamine seeking after extinction (Peters et al, 2008; Rocha and Kalivas, 2010). Additionally, reversible inactivation of the vmPFC decreases cue-induced methamphetamine but not cocaine seeking (McLaughlin and See, 2003; Rocha and Kalivas, 2010). We speculate that differences between mechanisms of cocaine $v s$ methamphetamine seeking are due to the different long-term effects of these drugs on glutamate transmission in corticostriatal circuits, which have a critical role in drug- and cue-induced reinstatement after extinction (Kalivas et al, 2009; Thomas et al, 2008) and incubation of drug craving (Loweth et al, 2014; Pickens et al, 2011; Wolf and Ferrario, 2010). For example, repeated exposure to cocaine but not amphetamine causes long-term alterations in NAc AMPA receptor surface expression (Boudreau et al, 2007; Boudreau and Wolf, 2005; Nelson et al, 2009). Additionally, lesions of $\mathrm{mPFC}$, a main glutamatergic projection to NAc (Voorn et al, 2004), decreases cocaine but not amphetamine locomotor sensitization (Tzschentke and Schmidt, 2000).

We found that reversible inactivation of the lateral OFC, previously shown to decrease 'incubated' heroin seeking after 14 withdrawal days (Fanous et al, 2012), had no effect on incubated methamphetamine seeking. These data, and previous findings (Pickens et al, 2011), suggest that mechanisms of incubation of opiate and psychostimulant craving are not identical. In this regard, we previously found that GDNF (glial cell line-derived neurotrophic factor) activity in ventral tegmental area is critical for incubation of cocaine but not heroin craving (Airavaara et al, 2011; Lu et al, 2009). In contrast, chronic delivery of the Toll-like receptor 4 antagonist $(+)$-naltrexone decreases incubation of heroin but not methamphetamine craving (Theberge et al, 2013). Our current and previous results are in agreement with the notion that different brain mechanisms mediate the motivational effects of opiates and psychostimulants and cues associated with these drug classe (Badiani, 2013; Badiani et al, 2011; Caprioli et al, 2009; Ettenberg, 2004, 2009).

Taken together, the available data suggest that dissociable neural substrates mediate incubation of drug craving for methamphetamine, heroin, and cocaine. However, this conclusion should be made with caution, because it is based on comparison across studies, which were performed at different times, with different cohorts of rats, and under different experimental conditions (eg, 9-h/day training sessions in the present study vs 6-h/day in our previous heroin and cocaine studies; Fanous et al, 2012; Koya et al, 2009).

\section{CONCLUSIONS}

Our anatomical mapping study has identified the CeA as a critical brain substrate for incubation of methamphetamine craving which together with previous results indicate that CeA neuronal activity is a common substrate for incubation of drug and non-drug reward craving. In contrast, our negative results for inactivation of different prefrontal cortex areas suggest that their role in incubation of craving after withdrawal is drug specific.

\section{FUNDING AND DISCLOSURE}

This work was supported by the Intramural Research Program of the National Institute on Drug Abuse. The authors declare no conflict of interest.

\section{REFERENCES}

Ahmed SH, Kenny PJ (2011). Cracking the molecular code of cocaine addiction. ILAR J 52: 309-320.

Airavaara M, Pickens CL, Stern AL, Wihbey KA, Harvey BK, Bossert JM et al (2011). Endogenous GDNF in ventral tegmental area and nucleus accumbens does not play a role in the incubation of heroin craving. Addict Biol 16: 261-272.

Alleweireldt AT, Hobbs RJ, Taylor AR, Neisewander JL (2006). Effects of SCH-23390 infused into the amygdala or adjacent cortex and basal ganglia on cocaine seeking and self-administration in rats. Neuropsychopharmacology 31: 363-374. 
Badiani A (2013). Substance-specific environmental influences on drug use and drug preference in animals and humans. Curr Opin Neurobiol 23: 588-596.

Badiani A, Belin D, Epstein DH, Calu DJ, Shaham Y (2011). Opiate versus psychostimulant addiction: the differences do matter. Nat Rev Neurosci 12: 685-700.

Balleine BW, Killcross S (2006). Parallel incentive processing: an integrated view of amygdala function. Trends Neurosci 29: 272-279.

Bossert JM, Ghitza UE, Lu L, Epstein DH, Shaham Y (2005). Neurobiology of relapse to heroin and cocaine seeking: an update and clinical implications. Eur J Pharmacol 526: 36-50.

Bossert JM, Marchant NJ, Calu DJ, Shaham Y (2013). The reinstatement model of drug relapse: recent neurobiological findings, emerging research topics, and translational research. Psychopharmacology 229: 453-476.

Boudreau AC, Reimers JM, Milovanovic M, Wolf ME (2007). Cell surface AMPA receptors in the rat nucleus accumbens increase during cocaine withdrawal but internalize after cocaine challenge in association with altered activation of mitogen-activated protein kinases. J Neurosci 27: 10621-10635.

Boudreau AC, Wolf ME (2005). Behavioral sensitization to cocaine is associated with increased AMPA receptor surface expression in the nucleus accumbens. J Neurosci 25: 9144-9151.

Calu DJ, Chen YW, Kawa AB, Nair SG, Shaham Y (2014). The use of the reinstatement model to study relapse to palatable food seeking during dieting. Neuropharmacology 76 Pt B: 395-406.

Caprioli D, Celentano M, Dubla A, Lucantonio F, Nencini P, Badiani A (2009). Ambience and drug choice: cocaine- and heroin-taking as a function of environmental context in humans and rats. Biol Psychiatry 65: 893-899.

Corbit LH, Balleine BW (2005). Double dissociation of basolateral and central amygdala lesions on the general and outcomespecific forms of pavlovian-instrumental transfer. J Neurosci 25: 962-970.

Counotte DS, Schiefer C, Shaham Y, O’Donnell P (2014). Timedependent decreases in nucleus accumbens AMPA/NMDA ratio and incubation of sucrose craving in adolescent and adult rats. Psychopharmacology 231: 1675-1684.

Doherty JM, Cooke BM, Frantz KJ (2013). A role for the prefrontal cortex in heroin-seeking after forced abstinence by adult male rats but not adolescents. Neuropsychopharmacology 38: 446-454.

Estes WK (1948). Discriminative conditioning; effects of a Pavlovian conditioned stimulus upon a subsequently established operant response. J Exp Psychol 38: 173-177.

Ettenberg A (2004). Opponent process properties of self-administered cocaine. Neurosci Biobehav Rev 27: 721-728.

Ettenberg A (2009). The runway model of drug self-administration. Pharmacol Biochem Behav 91: 271-277.

Everitt BJ, Parkinson JA, Olmstead MC, Arroyo M, Robledo P, Robbins TW (1999). Associative processes in addiction and reward. The role of amygdala-ventral striatal subsystems. Ann NY Acad Sci 877: 412-438.

Fanous S, Goldart EM, Theberge FR, Bossert JM, Shaham Y, Hope BT (2012). Role of orbitofrontal cortex neuronal ensembles in the expression of incubation of heroin craving. J Neurosci 32: 11600-11609.

Feltenstein MW, See RE (2008). The neurocircuitry of addiction: an overview. Br J Pharmacol 154: 261-274.

Fuchs RA, Branham RK, See RE (2006). Different neural substrates mediate cocaine seeking after abstinence versus extinction training: a critical role for the dorsolateral caudate-putamen. J Neurosci 26: 3584-3588.

Fuchs RA, Evans KA, Parker MP, See RE (2004). Differential involvement of orbitofrontal cortex subregions in conditioned cue-induced and cocaine-primed reinstatement of cocaine seeking in rats. J Neurosci 24: 6600-6610.
Grimm JW, Hope BT, Wise RA, Shaham Y (2001). Incubation of cocaine craving after withdrawal. Nature 412: 141-142.

Hall J, Parkinson JA, Connor TM, Dickinson A, Everitt BJ (2001). Involvement of the central nucleus of the amygdala and nucleus accumbens core in mediating Pavlovian influences on instrumental behaviour. Eur J Neurosci 13: 1984-1992.

Hiranita T, Nawata Y, Sakimura K, Anggadiredja K, Yamamoto T (2006). Suppression of methamphetamine-seeking behavior by nicotinic agonists. Proc Natl Acad Sci USA 103: 8523-8527.

Holland PC, Gallagher M (2003). Double dissociation of the effects of lesions of basolateral and central amygdala on conditioned stimulus-potentiated feeding and Pavlovian-instrumental transfer. Eur J Neurosci 17: 1680-1894.

Jaffe JH (1990). Drug addiction and drug abuse. In: Gilman AG, Rall TW, Nies AS, Taylor P (eds). Goodman \& Gilman's the Pharmacological Basis of Therapeutics. 8th edn. Pergamon Press: New York, NY, USA, pp 522-573.

Kalivas PW, Lalumiere RT, Knackstedt L, Shen H (2009). Glutamate transmission in addiction. Neuropharmacology 56(Suppl 1): 169-173.

Knackstedt LA, Moussawi K, Lalumiere R, Schwendt M, Klugmann M, Kalivas PW (2010). Extinction training after cocaine self-administration induces glutamatergic plasticity to inhibit cocaine seeking. J Neurosci 30: 7984-7992.

Koob GF, Le Moal M (2001). Drug addiction, dysregulation of reward, and allostasis. Neuropsychopharmacology 24: 97-129.

Koya E, Uejima JL, Wihbey KA, Bossert JM, Hope BT, Shaham Y (2009). Role of ventral medial prefrontal cortex in incubation of cocaine craving. Neuropharmacology 56: 177-185.

Krasnova IN, Marchant NJ, Ladenheim B, McCoy MT, Panlilio LV, Bossert JM et al (2014). Incubation of methamphetamine and palatable food craving after punishment-induced abstinence. Neuropsychopharmacology 39: 2008-2016.

Kruzich PJ, See RE (2001). Differential contributions of the basolateral and central amygdala in the acquisition and expression of conditioned relapse to cocaine-seeking behavior. J Neurosci 21: $\mathrm{RC} 155$.

Lee BR, Ma YY, Huang YH, Wang X, Otaka M, Ishikawa M et al (2013). Maturation of silent synapses in amygdala-accumbens projection contributes to incubation of cocaine craving. Nat Neurosci 16: 1644-1651.

Li C, Frantz KJ (2009). Attenuated incubation of cocaine seeking in male rats trained to self-administer cocaine during periadolescence. Psychopharmacology 204: 725-733.

Li YQ, Li FQ, Wang XY, Wu P, Zhao M, Xu CM et al (2008). Central amygdala extracellular signal-regulated kinase signaling pathway is critical to incubation of opiate craving. J Neurosci 28: $13248-13257$.

Lovibond PF (1983). Facilitation of instrumental behavior by a Pavlovian appetitive conditioned stimulus. J Exp Psychol Anim Behav Process 9: 225-247.

Loweth JA, Tseng KY, Wolf ME (2014). Adaptations in AMPA receptor transmission in the nucleus accumbens contributing to incubation of cocaine craving. Neuropharmacology $76 \mathrm{Pt} \mathbf{B}$ : 287-300.

Lu L, Grimm JW, Dempsey J, Shaham Y (2004a). Cocaine seeking over extended withdrawal periods in rats: different time courses of responding induced by cocaine cues versus cocaine priming over the first 6 months. Psychopharmacology 176: 101-108.

Lu L, Grimm JW, Hope BT, Shaham Y (2004b). Incubation of cocaine craving after withdrawal: a review of preclinical data. Neuropharmacology 47(Suppl 1): 214-226.

Lu L, Hope BT, Dempsey J, Liu SY, Bossert JM, Shaham Y (2005). Central amygdala ERK signaling pathway is critical to incubation of cocaine craving. Nat Neurosci 8: 212-219.

Lu L, Uejima JL, Gray SM, Bossert JM, Shaham Y (2007). Systemic and central amygdala injections of the mGluR(2/3) agonist 
LY379268 attenuate the expression of incubation of cocaine craving. Biol Psychiatry 61: 591-598.

Lu L, Wang X, Wu P, Xu C, Zhao M, Morales M et al (2009). Role of ventral tegmental area glial cell line-derived neurotrophic factor in incubation of cocaine craving. Biol Psychiatry 66: 137-145.

Ma YY, Lee BR, Wang X, Guo C, Liu L, Cui R et al (2014). Bidirectional modulation of incubation of cocaine craving by silent synapse-based remodeling of prefrontal cortex to accumbens projections. Neuron 83: 1453-1467.

Mahler SV, Berridge KC (2012). What and when to "want"? Amygdala-based focusing of incentive salience upon sugar and sex. Psychopharmacology 221: 407-426.

Marchant NJ, Li X, Shaham Y (2013). Recent developments in animal models of drug relapse. Curr Opin Neurobiol 23: 675-683.

McFarland K, Kalivas PW (2001). The circuitry mediating cocaineinduced reinstatement of drug-seeking behavior. J Neurosci 21: 8655-8663.

McLaughlin J, See RE (2003). Selective inactivation of the dorsomedial prefrontal cortex and the basolateral amygdala attenuates conditioned-cued reinstatement of extinguished cocaine-seeking behavior in rats. Psychopharmacology 168: 57-65.

Meil WM, See RE (1997). Lesions of the basolateral amygdala abolish the ability of drug associated cues to reinstate responding during withdrawal from self-administered cocaine. Behav Brain Res 87: 139-148.

Neisewander JL, Baker DA, Fuchs RA, Tran-Nguyen LT, Palmer A, Marshall JF (2000). Fos protein expression and cocaine-seeking behavior in rats after exposure to a cocaine self-administration environment. J Neurosci 20: 798-805.

Nelson CL, Milovanovic M, Wetter JB, Ford KA, Wolf ME (2009). Behavioral sensitization to amphetamine is not accompanied by changes in glutamate receptor surface expression in the rat nucleus accumbens. J Neurochem 109: 35-51.

O’Brien CP, Childress AR, McLellan AT, Ehrman R (1992). Classical conditioning in drug-dependent humans. Ann NY Acad Sci 654: 400-415.

Paxinos G, Watson C (2008). The Rat Brain in Stereotaxic Coordinates. Sixth edition. 3rd edn. Academic Press: San Diego, CA, USA.

Peters J, LaLumiere RT, Kalivas PW (2008). Infralimbic prefrontal cortex is responsible for inhibiting cocaine seeking in extinguished rats. J Neurosci 28: 6046-6053.

Pickens CL, Airavaara M, Theberge F, Fanous S, Hope BT, Shaham Y (2011). Neurobiology of the incubation of drug craving. Trends Neurosci 34: 411-420.

Pierce RC, Kumaresan V (2006). The mesolimbic dopamine system: the final common pathway for the reinforcing effect of drugs of abuse? Neurosci Biobehav Rev 30: 215-238.

Pockros LA, Pentkowski NS, Swinford SE, Neisewander JL (2011). Blockade of 5-HT2A receptors in the medial prefrontal cortex attenuates reinstatement of cue-elicited cocaine-seeking behavior in rats. Psychopharmacology 213: 307-320.

Rocha A, Kalivas PW (2010). Role of the prefrontal cortex and nucleus accumbens in reinstating methamphetamine seeking. Eur J Neurosci 31: 903-909.

Schmidt HD, Anderson SM, Famous KR, Kumaresan V, Pierce RC (2005). Anatomy and pharmacology of cocaine priming-induced reinstatement of drug seeking. Eur J Pharmacol 526: 65-76.
See RE (2002). Neural substrates of conditioned-cued relapse to drug-seeking behavior. Pharmacol Biochem Behav 71: 517-529.

Self DW (2004). Regulation of drug-taking and -seeking behaviors by neuroadaptations in the mesolimbic dopamine system. Neuropharmacology 47(Suppl 1): 242-255.

Shaham Y, Hope BT (2005). The role of neuroadaptations in relapse to drug seeking. Nat Neurosci 8: 1437-1439.

Shalev U, Grimm JW, Shaham Y (2002). Neurobiology of relapse to heroin and cocaine seeking: a review. Pharmacol Rev 54: 1-42.

Shalev U, Morales M, Hope B, Yap J, Shaham Y (2001). Timedependent changes in extinction behavior and stress-induced reinstatement of drug seeking following withdrawal from heroin in rats. Psychopharmacology 156: 98-107.

Shepard JD, Bossert JM, Liu SY, Shaham Y (2004). The anxiogenic drug yohimbine reinstates methamphetamine seeking in a rat model of drug relapse. Biol Psychiatry 55: 1082-1089.

Sutton MA, Schmidt EF, Choi KH, Schad CA, Whisler K, Simmons D et al (2003). Extinction-induced upregulation in AMPA receptors reduces cocaine-seeking behaviour. Nature 421: 70-75.

Theberge FR, Li X, Kambhampati S, Pickens CL St, Laurent R, Bossert JM et al (2013). Effect of chronic delivery of the Toll-like receptor 4 antagonist $(+)$-naltrexone on incubation of heroin craving. Biol Psychiatry 73: 729-737.

Thomas MJ, Kalivas PW, Shaham Y (2008). Neuroplasticity in the mesolimbic dopamine system and cocaine addiction. $\mathrm{Br} \mathrm{J}$ Pharmacol 154: 327-342.

Tzschentke TM, Schmidt WJ (2000). Differential effects of discrete subarea-specific lesions of the rat medial prefrontal cortex on amphetamine- and cocaine-induced behavioural sensitization. Cereb Cortex 10: 488-498.

Uejima JL, Bossert JM, Poles GC, Lu L (2007). Systemic and central amygdala injections of the mGluR2/3 agonist LY379268 attenuate the expression of incubation of sucrose craving in rats. Behav Brain Res 181: 292-296.

Van den Oever MC, Spijker S, Smit AB, De Vries TJ (2010). Prefrontal cortex plasticity mechanisms in drug seeking and relapse. Neurosci Biobehav Rev 35: 276-284.

Voorn P, Vanderschuren LJ, Groenewegen HJ, Robbins TW, Pennartz CM (2004). Putting a spin on the dorsal-ventral divide of the striatum. Trends Neurosci 27: 468-474.

Wikler A (1973). Dynamics of drug dependence. Implications of a conditioning theory for research and treatment. Arch Gen Psychiatry 28: 611-616.

Wise RA (2004). Dopamine, learning and motivation. Nat Rev Neurosci 5: 483-494.

Wise RA, Hoffman DC (1992). Localization of drug reward mechanisms by intracranial injections. Synapse 10: 247-263.

Wolf ME, Ferrario CR (2010). AMPA receptor plasticity in the nucleus accumbens after repeated exposure to cocaine. Neurosci Biobehav Rev 35: 185-211.

Wyvell CL, Berridge KC (2001). Incentive sensitization by previous amphetamine exposure: increased cue- triggered "wanting" for sucrose reward. J Neurosci 21: 7831-7840.

Yun IA, Nicola SM, Fields HL (2004). Contrasting effects of dopamine and glutamate receptor antagonist injection in the nucleus accumbens suggest a neural mechanism underlying cue-evoked goal-directed behavior. Eur J Neurosci 20: 249-263.

Supplementary Information accompanies the paper on the Neuropsychopharmacology website (http://www.nature.com/npp) 